\title{
Revista Colombiana de

\section{Alternativas terapéuticas al manejo farmacológico con estatinas en adultos con dislipidemia. Revisión sistemática de la literatura y recomendaciones generales}

\author{
Ángel A. García ${ }^{\mathrm{a}, \mathrm{b}, *}$, Óscar Múñoz ${ }^{\mathrm{a}, \mathrm{c}}$, Daniel G. Fernández ${ }^{\mathrm{a}, \mathrm{b}}$, \\ Angélica M. Higuera ${ }^{a, b}$, Álvaro J. Ruiz ${ }^{d}$, Pablo A. Montoya ${ }^{e}$, Juan M. Toro ${ }^{f}$, \\ Juan M. Arteaga ${ }^{\mathrm{g}}$, Alonso M. Villamizar ${ }^{\mathrm{h}}$, Gregorio Sánchez ${ }^{\mathrm{i}, \mathrm{j}}$, y Yadira Villalba ${ }^{k}$
}

a Grupo desarrollador de la Guía de Práctica Clínica para la Prevención, Detección Temprana, Diagnóstico, Tratamiento y Seguimiento de las Dislipidemias en población mayor de 18 años

b Unidad de Cardiología, Departamento de Epidemiología Clínica y Bioestadística, Hospital Universitario de San Ignacio, Pontificia Universidad Javeriana, Bogotá, Colombia

c Unidad de Clínica Hospitalaria, Departamento de Epidemiología Clínica y Bioestadística, Hospital Universitario de San Ignacio, Pontificia Universidad Javeriana, Bogotá, Colombia

d Departamento de Medicina Interna, Departamento de Epidemiología Clínica y Bioestadística, Pontificia Universidad Javeriana, Bogotá, Colombia

e Departamento de Medicina Interna, Pontificia Universidad Javeriana, Hospital Universitario San Ignacio, Bogotá, Colombia

f Departamento de Medicina Interna, Universidad de Antioquia, Hospital Pablo Tobón Uribe, Medellín, Antioquia, Colombia

s Departamento de Medicina Interna, Universidad Nacional de Colombia, Bogotá, Colombia

h Sociedad Colombiana de Cardiología y Cirugía Cardiovascular, Fundación Clínica Shaio, Bogotá, Colombia

' Asociación Colombiana de Medicina Interna

j Hospital Departamental Universitario del Quindío San Juan de Dios, Quindío, Colombia

k Asociación Colombiana de Endocrinología, Diabetes y Metabolismo, Universidad de Cartagena, Cartagena, Colombia

Recibido el 13 de octubre de 2014; aceptado el 18 de abril de 2015

Disponible en Internet el 6 de junio de 2015

\section{PALABRAS CLAVE \\ Enfermedad \\ cardiovascular; \\ Hipercolesterolemia; Lípidos; \\ Tratamiento; \\ Estatinas}

\begin{abstract}
Resumen
Objetivo: Evaluar el impacto sobre la incidencia y recurrencia de eventos cardiovasculares y cerebrovasculares (prevención primaria y secundaria), los niveles de las fracciones lipídicas y la incidencia de efectos secundarios en personas con hipercolesterolemia intolerantes al tratamiento con las estatinas.

Métodos: Se elaboró una guía de práctica clínica siguiendo los lineamientos de la guía metodológica del Ministerio de Salud y Protección Social para recolectar de forma sistemática la evidencia científica y formular las recomendaciones utilizando la metodología GRADE.
\end{abstract}

\footnotetext{
* Autor para correspondencia.

Correo electrónico: angel.garcia@javeriana.edu.co (Á.A. García).
} 
Población: Población adulta, con diagnóstico de dislipidemia, o en riesgo de desarrollarla, intolerantes al manejo con las estatinas.

Recomendaciones: Se formulan recomendaciones a favor del uso de diferentes medicamentos (los fibratos, la niacina, los ácidos omega 3, las resinas secuestradoras de ácidos biliares, el ezetimibe), según su efectividad hipolipemiante y reducción de eventos cardiovasculares, como propuesta adaptada al contexto social colombiano.

(C) 2014 Sociedad Colombiana de Cardiología y Cirugía Cardiovascular. Publicado por Elsevier España, S.L.U. Este es un artículo Open Access bajo la licencia CC BY-NC-ND (http://creativecommons.org/licenses/by-nc-nd/4.0/).

Therapeutic alternatives to pharmacological management with statin drugs in adults with dyslipidemia. Systematic literature review and general recommendations

\begin{abstract}
Objective: To evaluate the impact on the incidence and recurrence of cardiovascular and cerebrovascular events (primary and secondary prevention), the levels of lipid fractions and the incidence of side effects in people with hypercholesterolemia intolerant to statin therapy. Methods: A clinical practice guideline was developed following the guidelines of the methodological guidance of the Ministry of Health and Social Protection to systematically collect the evidence and make recommendations using the GRADE methodology.

Population: Adult population, diagnosed with dyslipidemia or at risk of developing, intolerant to management with statin drugs.

Recommendations: Recommendations for the use of different medications (fibrates, niacin, omega 3 acids, bile acid binding resins, ezetimibe) are formulated according to their lipidlowering effectiveness and reduction of cardiovascular events, a proposal adapted to the Colombian social context.

(C) 2014 Sociedad Colombiana de Cardiología y Cirugía Cardiovascular. Published by Elsevier España, S.L.U. This is an open access article under the CC BY-NC-ND license (http://creativecommons.org/licenses/by-nc-nd/4.0/).
\end{abstract}

\section{Introducción}

El pilar fundamental del tratamiento farmacológico de las dislipidemias es la administración de las estatinas, que se seleccionan y dosifican según el riesgo de desarrollar eventos cardiacos y cerebrovasculares a 10 años, y teniendo en cuenta condiciones clínicas específicas y la historia de eventos cardiocerebrovasculares previos. Las guías de práctica clínica de grupos internacionales publicadas en los últimos dos años han actualizado las recomendaciones de manejo farmacológico de las dislipidemias, dejando a las estatinas como la terapia de elección en este grupo de pacientes ${ }^{1-5}$. Esta recomendación está soportada de forma amplia en evidencia científica sólida, que ha confirmado el beneficio en prevención primaria y secundaria en diferentes grupos poblacionales.

Las recomendaciones sobre otras opciones de manejo farmacológico en pacientes intolerantes a las estatinas son, sin embargo, muy limitadas. A continuación, se presenta la revisión de la literatura, discusión y recomendaciones acerca de estas opciones de tratamiento farmacológico ${ }^{6-10}$.

El Ministerio de Salud y Protección Social ha encargado a la Pontificia Universidad Javeriana y a la Alianza CINETS, conformada por las Universidades Javeriana, Nacional y Antioquia el desarrollo de una Guía Clínica Sobre la Prevención, Detección Temprana, Diagnóstico, Tratamiento y Seguimiento de las Dislipidemias en la Población Mayor de 18 Años. El resultado de ese proceso, en lo referente a las alternativas terapéuticas al manejo farmacológico con las estatinas en adultos con dislipidemia, se presenta de forma resumida en el presente escrito. Cabe resaltar que en este proceso también participaron activamente la Asociación Colombiana de Medicina Interna, la Sociedad Colombiana de Cardiología y la Asociación Colombiana de Endocrinología.

\section{Métodos}

El proceso de desarrollo de la guía se describe detalladamente en el Manual para desarrollo de guías de práctica clínica y en la actualización de dicha metodología (disponible en la página web del Ministerio de Salud y Protección Social) ${ }^{11}$.

Cabe resaltar que dicha metodología facilita la búsqueda sistemática de la evidencia científica (que incluya tanto revisiones sistemáticas de la literatura como estudios primarios). Así mismo establece una metodología clara para la selección de la evidencia que se va a utilizar, después de una cuidadosa evaluación de la calidad de la misma. La versión completa de la guía, en la que se presentan los resultados de todas estas evaluaciones puede ser consultada en la página web del Ministerio de Salud y Protección Social, así como en la página web de la alianza CINETS. 
El grupo metodológico preparó un resumen de la evidencia disponible y lo presentó al panel completo durante las reuniones de generación de recomendaciones. Este panel incluyó a representantes de diversas especialidades médicas (los cardiólogos, los endocrinólogos, los médicos internistas, los médicos de familia y los médicos generales), así como a otros profesionales de la salud (los nutricionistas y los terapeutas físicos). Cabe resaltar que durante todo el proceso se contó adicionalmente con representantes de los pacientes, que aportaron activamente en la generación de recomendaciones. Todos los integrantes del panel presentaron abiertamente la declaración de sus conflictos de intereses. Estos documentos están disponibles en la versión completa de la guía.

Durante las reuniones de generación de recomendaciones se siguió la metodología propuesta por el grupo GRADE, que permite tener en cuenta no solo la calidad de la evidencia disponible, sino las consideraciones de costos, las preferencias de los pacientes y la relación entre los beneficios y los riesgos de las tecnologías de interés (las pruebas, las estrategias de manejo, las intervenciones y los medicamentos). Las recomendaciones presentadas muestran por tanto la fuerza de la recomendación (fuerte o débil), la dirección de la misma (a favor o en contra de la intervención) así como la calidad de la evidencia que la soporta (muy baja, baja, moderada, alta o consenso de expertos).

La versión final de la guía fue evaluada por pares internacionales, seleccionados por el Ministerio de la Protección Social, expertos tanto en dislipidemia como en metodología. Sus aportes fueron tenidos en cuenta por el grupo desarrollador de la guía.

\section{Resultados}

\section{Fibratos}

El metaanálisis de Jun ${ }^{6}$, evaluó el impacto del tratamiento con los fibratos frente al placebo sobre los desenlaces cardiovasculares, e incluyó a un total de 45.058 participantes, con un tiempo de seguimiento que varió desde un año hasta ocho años. No se encontraron diferencias estadísticamente significativas en los desenlaces de mortalidad global o cardiovascular. En cuanto a los niveles de fracciones lipídicas, se observó una reducción significativa en los niveles de los triglicéridos y un discreto cambio en los niveles del colesterol LDL y en el colesterol total. El estudio de Abourbih ${ }^{7}$, que se seleccionó para evaluar el desenlace de infarto agudo del miocardio, analizó el efecto del tratamiento con los fibratos frente al placebo, en 25.655 participantes con predominio de dislipidemia mixta, con un tiempo de seguimiento entre ocho y 322 semanas; se encontraron diferencias clínica y estadísticamente significativas, con una disminución del $22 \%$ en los eventos de infarto agudo de miocardio asociado con el tratamiento con los fibratos (OR 0,78, IC 95\% 0,69; 0,89). El metaanálisis de Zhou ${ }^{8}$, evaluó el impacto del tratamiento con los fibratos en la prevención de ACV en comparación con placebo. Se incluyó a 37.791 participantes, que fueron seguidos por un período de tiempo desde 30 hasta 104 meses. Se encontró que el tratamiento con los fibratos no tuvo ningún efecto sobre el riesgo de presentar ACV.
Para analizar el impacto de la terapia combinada de los fibratos con las estatinas frente a la monoterapia con las estatinas en adultos se eligió el estudio de Sharma ${ }^{9}$, no obstante, el tiempo de seguimiento de los pacientes fue muy corto (entre 12 y 18 semanas) y el número de eventos de infarto agudo del miocardio y de fallecimiento fue muy escaso, lo cual no permitió evaluar si existen diferencias significativas. Tampoco se encontraron diferencias significativas en cuanto a los niveles de fracciones lipídicas. Sin embargo, la administración de esta terapia en paciente con hipertrigliceridemia con valores superiores a $500 \mathrm{mg} / \mathrm{dl}$ disminuyó el riesgo de presentación de pancreatitis, sin impacto sobre otros desenlaces de interés.

\section{Niacina}

La revisión sistemática de la literatura de Lavigne and Karas ${ }^{10}$, evaluó el uso de la niacina en la prevención primaria y secundaria de eventos cardiovasculares, tanto sola como asociada con el uso de las estatinas, con un mínimo de seguimiento de seis meses. Se encontró una reducción significativa de los eventos cardiovasculares (muerte de origen cardiovascular, infarto agudo del miocardio no fatal, hospitalización por cualquier síndrome coronario agudo, ataque cerebrovascular y revascularización miocárdica por cualquier método) en el grupo tratado con niacina (OR 0,66; IC 95\% 0,49; 0,89. I2 59\%). También se encontró una reducción significativa de los eventos coronarios mayores (infarto agudo del miocardio no fatal, muerte de origen cardiovascular y ataque cerebrovascular isquémico o hemorrágico) (OR 0,75 ; IC 95\% 0,59; 0,96).

Cuando se evaluó el uso de la niacina en conjunto con las estatinas, se encontró una diferencia significativa para el desenlace de eventos cardiovasculares (OR 0,31; IC 95\% $0,1-0,97 ; p<0,04)$. Para evaluar el desenlace de mortalidad se seleccionó el metaanálisis de Duggal ${ }^{11}$, que incluyó a 5.137 pacientes con historia de IAM o de enfermedad coronaria, con un período de seguimiento de por lo menos un año. Las dosis utilizadas de la niacina fueron de $0,5 \mathrm{~g} /$ día a $3 \mathrm{~g} /$ día, en fórmulas de liberación prolongada. Se encontró reducción de la necesidad de revascularización miocárdica (RR 0,30; IC 95\% 0,15; 0,62), para infarto agudo del miocardio no fatal (RR 0,719; IC 95\% 0,603; 0,856), y para ataque cerebrovascular y ataque isquémico transitorio (RR 0,759 ; IC $95 \% 0,613 ; 0,940)$. No se encontró diferencia para mortalidad por todas las causas o muerte cardiovascular.

El Grupo HPS2-THRIVE ${ }^{12}$, evaluó el papel de la niacina en la prevención de eventos cardiovasculares en pacientes de alto riesgo cardiovascular bajo tratamiento con la estatina, a dosis de $2 \mathrm{~g} /$ día más laropiprant $40 \mathrm{mg} /$ día (antagonista del DP1, para disminución de efectos secundarios; principalmente el rubor), en 25.673 pacientes en manejo con la estatina y con diagnóstico de enfermedad arterial oclusiva. El seguimiento promedio fue de un año; la disminución promedio de las fracciones lipídicas en el grupo de intervención fue de $12,9 \mathrm{mg} / \mathrm{dL}$ para LDL, y de $9,88 \mathrm{mg} / \mathrm{dL}$ para triglicéridos, con aumento de $6,8 \mathrm{mg} / \mathrm{dL}$ para $\mathrm{HDL}$; sin embargo, dicha evaluación se llevó a cabo durante el período prealeatorización, $y$, dada la terminación precoz del estudio, los autores se limitan en la discusión del artículo a hacer un estimativo del comportamiento a lo largo de los tres años. 
El estudio fue detenido por el aumento de los efectos secundarios (principalmente, en la piel) $(5,4$ vs. $1,2 \%$, exceso de eventos del $4,2 \%$, con una desviación estándar de $0,2 \%$. $p<0,0001$ ), entre los cuales se incluyeron el prurito, la exantema y el rubor. Los efectos gastrointestinales también fueron significativamente mayores en el grupo de intervención ( 3,9 vs. $1,7 \%$, exceso de eventos del $2,1 \%$ con DE $0,2 \% . p<0,0001)$, entre los cuales los más frecuentes fueron sensación de indigestión y diarrea.

Sharma ${ }^{9}$, incluyó en su revisión principalmente a la población que requería terapia intensiva hipolipemiante (alto riesgo cardiovascular o prevención secundaria), en tratamiento con la niacina más la estatina vs. la estatina en monoterapia, y no encontró diferencias significativas para mortalidad por todas las causas, muerte por causa vascular e infarto agudo del miorcardio (OR 4,64; IC 95\% 0,08; 283,78 ); tampoco para síndrome coronario agudo (incluye infarto agudo de miorcardio y angina inestable) (OR 0,91; IC $95 \% 0,12 ; 6,62)$, ni para cualquier tipo de ataque cerebrovascular (incluía hemorrágico e isquémico) (OR 0,12; IC 95\% $0 ; 6,21)$ y tampoco para eventos adversos serios (OR 1,29; IC 95\% 0,44; 3,80).

Como principales limitantes de los estudios descritos, cabe mencionar: el momento histórico de la realización de los estudios primarios (coadministracion de múltiples medicamentos con impacto en eventos cardiovasculares (los bloqueadores beta, los IECA, la aspirina, etc.), múltiples dosis y tipo de la estatina y el manejo e intervenciones efectivas sobre la enfermedad coronaria diferentes a la niacina), y la falta de análisis específicos para el uso de la niacina con diferentes dosis de la estatina. Por estas razones y a la luz de los avances actuales en el tratamiento del síndrome coronario agudo, la información es limitada y quizás controversial.

\section{Omega 3}

La revisión de $\operatorname{Rizos}^{13}$, evaluó el impacto de la administración del omega 3 (a través de la dieta o por medio de suplementos) en pacientes con eventos cardiovasculares o cerebrovasculares previos (prevención secundaria), con una dosis promedio de $1,5 \mathrm{~g} /$ día, en 28.559 pacientes, con un tiempo promedio de seguimiento de dos años. No se evidenciaron diferencias clínica ni estadísticamente significativas en la incidencia de muerte por cualquier causa, IAM o ACV. Se halló una diferencia estadísticamente significativa en la tasa de muerte de origen coronario (RR 0,81; IC 95\% 0,70; $0,93)$. El estudio de Roncaglioni ${ }^{14}$ evaluó a 125 pacientes con múltiples factores de riesgo cardiovascular, pero sin IAM previo (prevención primaria) que fueron aleatorizados a omega 3 o placebo (aceite de oliva). La dosis fue de $1 \mathrm{~g} /$ día y el tiempo de seguimiento fue, en promedio, de cinco años. En esta población no se hallaron diferencias clínica ni estadísticamente significativas en la incidencia de mortalidad de origen cardiovascular e infarto agudo del miocardio. La revisión sistemática de Chowdhury ${ }^{15}$, evaluó la incidencia de ACV (isquémicos o hemorrágicos) en pacientes que recibieron suplementación con omega-3 como prevención primaria, sin encontrar diferencia en este desenlace.

La revisión de Hooper ${ }^{16}$, evaluó el impacto de los suplementos de omega 3 en las fracciones lipídicas. Se hizo un análisis de subgrupos para las diferentes dosis de omega 3 , y se mostró que la suplementación de omega 3 reduce, de forma clínica y estadísticamente significativa, los niveles de triglicéridos, con un efecto mayor cuanta más alta sea la dosis administrada. De igual forma, se evidencia un leve aumento, estadística, pero no clínicamente significativo en los niveles de colesterol LDL. No se documentó impacto alguno en los niveles de colesterol total ni de colesterol HDL en ninguna de las dosis evaluadas. Este mismo estudio evaluó, además, la incidencia de efectos secundarios, y encontró un aumento de más de tres veces en la incidencia de mal olor o de sabor a pescado, que resulta significativo, tanto clínica como estadísticamente. Esto condiciona la falta de adherencia al tratamiento. Los pacientes en suplementación de omega 3 suspendieron la terapia un $62 \%$ más que los pacientes con placebo (RR 1,62; IC 95\% 1, 10; $2,40)$. La revisión sistemática publicada por $\operatorname{Pan}^{17}$, evaluó la evidencia disponible, hasta el momento, sobre el impacto del consumo del omega 3 de origen vegetal, y, específicamente, el consumo de ácido linolénico, en la incidencia de eventos cardiovasculares y cerebrovasculares. La revisión no identificó ningún experimento clínico aleatorizado, por lo cual las conclusiones se basan en estudios de cohortes. Los resultados son similares a los presentados para los estudios de suplementación del omega 3 de origen marino, observando una disminución clínica y estadísticamente significativa en la incidencia de los fallecimientos de origen cardiovascular (RR 0,80; IC 95\% 0,68; 0,98), sin evidencia de impacto sobre la incidencia de infarto agudo del miocardio no fatal, ni de ataque cerebrovascular.

\section{Resinas secuestradoras de ácidos biliares}

Los resultados sobre el uso de las resinas secuestradoras de ácidos biliares son heterogéneos, por lo cual no fue posible hacer un metaanálisis de la información; no obstante, los estudios de Davidson ${ }^{18}$, Gordon ${ }^{19}$, Rifkin ${ }^{20}$, Dorr $^{21}$, Insull ${ }^{22}$, mostraron que no hay diferencias estadísticamente significativas respecto a los desenlaces de muerte y de infarto agudo del miocardio, con reducción clínica y estadísticamente significativa en los niveles del colesterol LDL, y con ligera elevación de los niveles de los triglicéridos.

La incidencia de efectos secundarios fue significativamente más alta entre los pacientes que recibieron las resinas secuestradoras de ácidos biliares que entre quienes recibieron el placebo. El efecto secundario más común fue el estreñimiento. Dichos efectos secundarios conllevaron una mayor probabilidad de suspender el medicamento.

Para evaluar la efectividad de las resinas secuestradoras de ácidos biliares asociadas con el uso de las estatinas se seleccionó la revisión de Sharma9 ${ }^{9}$, que comparó el uso de las resinas secuestradoras de ácidos biliares (colestiramina 1,6-2,4 g/día, colesevelam 2,3-3,8 g/día, colestipol 1,65-20 g/día), con la administración de las estatinas en monoterapia. Se incluyó a una población total de 511 pacientes que fue seguida durante 24 semanas. Se halló muy escasa evidencia evaluando el impacto sobre mortalidad total y para infarto agudo del miocardio, sin diferencias estadísticamente significativas. También se encontró una discreta reducción del LDL en la terapia combinada en comparación 
con la monoterapia, entre 3 y $10 \%$ de los niveles de LDL inicial.

\section{Ezetimibe}

La revisión sistemática de la literatura de Pandor $^{23}$ evaluó el uso del ezetimibe $10 \mathrm{mg} /$ día frente a placebo, en 2.722 pacientes mayores de 18 años con diagnóstico de hipercolesterolemia primaria, que tenían alto riesgo de enfermedad cardiovascular, con un tiempo de seguimiento de 12 semanas. No se reportó información respecto a incidencia de mortalidad, infarto agudo del miocardio o ataque cerebrovascular. En cuanto a los niveles de fracciones lipídicas se evidenció una disminución clínica y estadísticamente significativa en el valor del colesterol LDL (porcentaje medio de cambio $-18,58 \%$ IC $95 \%-19,67 ;-17,48$ ) y el colesterol total (porcentaje medio de cambio -13,46\% IC 95\% -14,22; $-12,70)$. El cambio en el cHDL y en los triglicéridos fue estadísticamente significativo, pero no logra significación clínica.

La revisión de Sharma9 ${ }^{9}$, evaluó el uso del ezetimibe asociado con las estatinas (la simvastatina o la atorvastatina), en dosis bajas y altas, en un total de 13.463 pacientes mayores de 18 años con diagnóstico de hipercolesterolemia. No se hallaron diferencias estadísticamente significativas en cuanto a la administración del ezetimibe en combinación con las estatinas en la incidencia de mortalidad, mortalidad vascular o efectos adversos.

Para evaluar el impacto sobre los niveles de las fracciones lipídicas se seleccionó el metaanálisis de Mikhailidis ${ }^{24}$, que incluyó a 5.080 pacientes, con un tiempo de seguimiento promedio de 14,8 semanas. Se encontró una disminución clínica y estadísticamente significativa en el valor del colesterol total (porcentaje medio de cambio -10,01\% IC 95\% $-11,52 ;-8,49)$ y del colesterol LDL (porcentaje medio de cambio $-14,11 \%$ IC $95 \%-16,13 ;-12,10)$, aunque se halló gran heterogeneidad en estos resultados $\left(1^{2}\right.$ de $65,8 \%$ $(p=0,001)$ y de $68,4 \%(p<0,001)$, respectivamente).

\section{Discusión}

Las alternativas terapéuticas para el manejo de la dislipidemia como segunda línea de manejo después de las estatinas o para pacientes intolerantes a estas son reducidas y la evidencia sobre su efectividad clínica e impacto sobre desenlaces duros como mortalidad o eventos cardiovasculares es limitada. Encontrando en general un mínimo efecto. Adicionalmente llama la atención el incremento de eventos adversos, que disminuye la adherencia a este tipo de terapia, así como por el pobre impacto sobre las diferentes fracciones lipídicas.

Como limitante de los estudios primarios y revisiones sistemáticas descritas, la gran mayoría de los artículos fueron realizados en las décadas de los años 80 y 90, momento histórico en el cual no se utilizaban de forma rutinaria en el manejo de los pacientes con enfermedad cardiovascular medicamentos como los antiagregantes, los anticoagulantes y las estatinas; también una importante heterogeneidad respecto al perfil de riesgo cardiovascular y a las consideraciones de prevención primarios y secundarios en las cohortes de seguimiento.
Por esta razón, las guías de práctica clínica nacionales e internacionales dirigen sus esfuerzos y recomendaciones a mantener la terapia con las estatinas y en caso de intolerancia considerar la disminución de la dosis y al cambio del tipo de la estatina, antes de recomendar el uso de otras alternativas terapéuticas.

Sin embargo, una vez que el personal de salud encargado del tratamiento de este grupo de pacientes determina que existe intolerancia a las estatinas, existe un gran vacío del conocimiento. En relación con las diferentes opciones de manejo, en términos generales, con la evidencia anteriormente descrita, se considera que son razonables como segunda línea de manejo los fibratos o la niacina, en una tercera línea de manejo los ácidos omega 3 y quizás para casos especiales y bajo manejo especializado, se puede considerar el uso de las resinas de secuestradoras de ácidos biliares. En el momento de la realización de la presente guía, no se encontró evidencia que permitiera recomendar escenarios específicos para la administración del ezetimibe o administración de las estatinas en combinación con algunas de las terapias anteriormente mencionadas como manejo rutinario del paciente adulto con dislipidemia. Sin embargo, posterior a la publicación de esta guía en el año 2014, se ha publicado nueva información sobre estudios clínicos, en particular sobre el uso de la simvastatina en combinación con el ezetimibe (IMPROVE-IT: Examining Outcomes in Subjects With Acute Coronary Syndrome: Vytorin (Ezetimibe/Simvastatin) vs. Simvastatin NCT00202878) $)^{25}$, con resultados prometedores que deberán ser incluidos en las próximas guías de manejo para la dislipidemia, sin embargo, sus resultados muestran beneficio de la administración, de la combinación y no fue evaluada la administración del ezetimibe como monoterapia. Así mismo, se encuentran en desarrollo un novedoso grupo de medicamentos (PCSK9 por inhibits proprotein convertase subtilisin-kexin type 9), que logran una importante reducción de los niveles de LDL y se proyectan como potenciales alternativas de manejo en monoterapia o en combinación con las estatinas, sin embargo, actualmente, se encuentran en desarrollo estudios en la fase 2 y 3 de la investigación.

\section{Conclusiones}

A continuación se presentan las recomendaciones finales de la Guía de práctica clínica para la prevención, detección temprana, diagnóstico, tratamiento y seguimiento de las dislipidemias, en cuanto al tratamiento farmacológico con las estatinas. Dichas recomendaciones fueron derivadas de la evidencia presentada, y las consideraciones mostradas en la discusión. La información completa del proceso para generar las recomendaciones se encuentra en la versión completa de la Guía disponible a través de la página electrónica del Ministerio de Salud y Protección Social y en la página electrónica de la Alianza CINETS (fig. 1. Alternativas de tratamiento farmacológico).

\section{Recomendaciones sobre los fibratos}

1. En personas con hipertrigliceridemia mayor o igual a $500 \mathrm{mg} / \mathrm{dL}$ se sugiere iniciar el tratamiento farmacológico con los fibratos y estudiar la causa de base. 


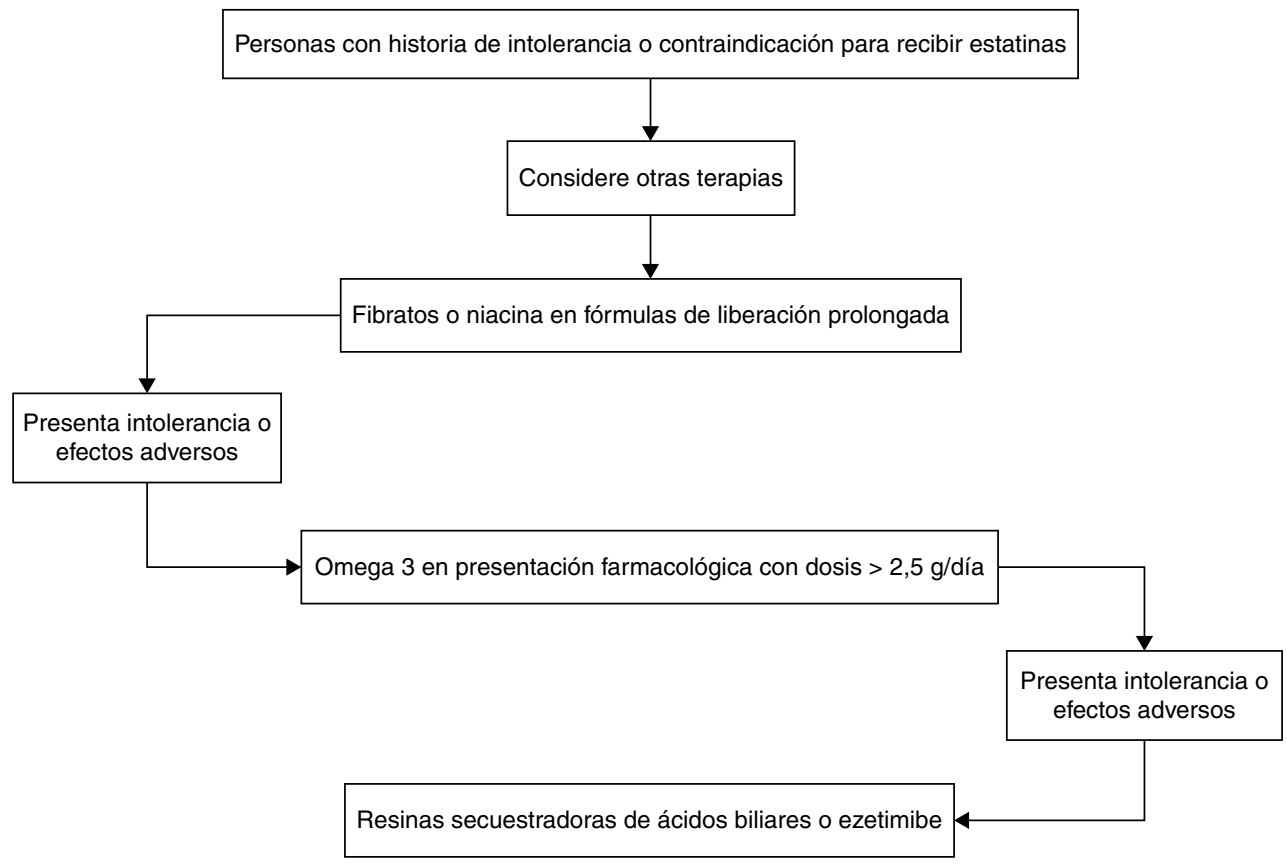

Figura 1 Alternativas de tratamiento farmacológico.

Recomendación débil a favor de la intervención. Calidad de la evidencia: $\oplus \oplus \oplus \ominus$ moderada.

2. No se recomienda el uso de los fibratos como primera línea de tratamiento para personas con dislipidemia mixta cuando los niveles de triglicéridos sean menores de $500 \mathrm{mg} / \mathrm{dL}$. Recomendación fuerte en contra de la intervención. Calidad de la evidencia: $\oplus \oplus \oplus \ominus$ moderada.

3. Se sugiere el uso de los fibratos en personas que presenten dislipidemia mixta con predominio de hipertrigliceridemia (menor de $500 \mathrm{mg} / \mathrm{dL}$ ) y que presenten intolerancia o contraindicación para el uso de las estatinas. Recomendación débil a favor de la intervención. Calidad de la evidencia: $\oplus \oplus \oplus \ominus$ moderada.

4. No se sugiere usar la terapia combinada de los fibratos con las estatinas como primera línea de tratamiento para la dislipidemia mixta con predominio de la hipertrigliceridemia. Recomendación débil en contra de la intervención. Calidad de la evidencia: $\oplus \oplus \ominus \ominus$ baja.

\section{Recomendaciones sobre el ácido nicotínico (niacina)}

1. En la población adulta con dislipidemia mixta no se recomienda el uso del ácido nicotínico como primera línea de tratamiento para la dislipidemia. Recomendación fuerte en contra de la intervención. Calidad de la evidencia: $\oplus \oplus \ominus \ominus$ baja.

2. En personas con dislipidemia e historia de intolerancia o contraindicación para recibir las estatinas, se sugiere considerar el uso del ácido nicotínico en dosis de $0,5 \mathrm{~g} /$ día a $3 \mathrm{~g} / \mathrm{día}$, en fórmulas de liberación prolongada como terapia alternativa. Recomendación débil a favor de la intervención. Calidad de la evidencia: $\oplus \oplus \ominus \ominus$ baja.

3. En la población adulta con dislipidemia mixta no se sugiere utilizar la combinación del ácido nicotínico y las estatinas como primera línea de manejo en personas con dislipidemia. Recomendación débil en contra de la intervención. Calidad de la evidencia: $\oplus \oplus \oplus \ominus$ moderada.

\section{Recomendaciones sobre el omega 3}

1. En la población adulta con dislipidemia mixta no se recomienda el uso del omega 3 como primera línea de tratamiento para la dislipidemia. Recomendación fuerte en contra de la intervención. Calidad de la evidencia: $\oplus \oplus \oplus \ominus$ moderada.

2. En la población adulta con dislipidemia mixta se sugiere administrar suplementos del omega 3 (presentación farmacológica con dosis superiores a 2,5 g/día) en pacientes con eventos previos (prevención secundaria) que tengan intolerancia o contraindicación para recibir las estatinas, los fibratos y el ácido nicotínico. Recomendación débil a favor de la intervención. Calidad de la evidencia: $\oplus \oplus \oplus \ominus$ moderada.

3. En personas con las características previamente mencionadas, y que, adicionalmente, declaren no estar dispuestas a aceptar el efecto adverso (mal olor o sabor a pescado), se sugiere considerar el uso de otros medicamentos, como el ezetimibe o las resinas secuestradoras de ácidos biliares. Recomendación débil a favor de la intervención. Calidad de la evidencia: consenso de expertos.

4. Se sugiere administrar suplementos del omega 3 (presentación farmacológica con dosis superiores a 2,5 g/día) en pacientes con hipertrigliceridemia persistente, mayor de $500 \mathrm{mg} / \mathrm{dL}$, a pesar del manejo con los fibratos. Recomendación débil a favor de la intervención. Calidad de la evidencia: $\oplus \oplus \oplus \oplus$ alta.

5. En la población adulta con dislipidemia mixta no se sugiere utilizar la combinación del omega 3 y las 
estatinas. Recomendación débil en contra de la intervención. Calidad de la evidencia: $\oplus \oplus \ominus \ominus$ baja.

\section{Puntos de buena práctica clínica}

- Se insiste en que se está haciendo referencia al omega3 en presentación farmacológica, y no a dietas ricas en omega 3 ni en omega 3 de origen vegetal. Dichas formas de administración no proporcionan las dosis que han demostrado beneficios en los estudios clínicos presentados.

- De igual forma, cabe resaltar que la mayoría de los suplementos nutricionales disponibles en el mercado presentan concentraciones del omega 3 de entre $300 \mathrm{mg}$ y $720 \mathrm{mg}$, por lo cual se requerirían, como mínimo, tres cápsulas de este tipo, y habitualmente ocho, para alcanzar la dosis sugerida de $2,5 \mathrm{~g} /$ día, como mínimo.

\section{Recomendaciones sobre resinas}

1. En la población adulta con hipercolesterolemia no se recomienda el uso de resinas secuestradoras de ácidos biliares como primera línea de tratamiento para la dislipidemia. Recomendación fuerte en contra de la intervención. Calidad de la evidencia: $\oplus \oplus \oplus \ominus$ moderada.

2. En la población adulta con hipercolesterolemia se sugiere considerar el uso de las resinas secuestradoras de ácidos biliares en monoterapia como terapia alterna en caso de intolerancia a otras terapias. Recomendación débil a favor de la intervención. Calidad de la evidencia: $\oplus \ominus \ominus \ominus$ muy baja.

\section{Recomendaciones sobre el ezetimibe}

1. En la población adulta con hipercolesterolemia no se recomienda el uso del ezetimibe como primera línea de tratamiento para la dislipidemia. Recomendación fuerte en contra de la intervención. Calidad de la evidencia: $\oplus \oplus \oplus \ominus$ moderada.

2. En pacientes adultos con hipercolesterolemia con intolerancia a otras terapias o efectos adversos con estas, se puede considerar el uso del ezetimibe en monoterapia. Recomendación débil a favor de la intervención. Calidad de la evidencia: $\oplus \oplus \oplus \ominus$ moderada.

3. En la población adulta con hipercolesterolemia no se recomienda el uso del ezetimibe en combinación con las estatinas. Recomendación débil en contra de la intervención. Calidad de la evidencia: $\oplus \ominus \ominus \ominus$ muy baja.

\section{Responsabilidades éticas}

Protección de personas y animales. Los autores declaran que para esta investigación no se han realizado experimentos en seres humanos ni en animales.

Confidencialidad de los datos. Los autores declaran que en este artículo no aparecen datos de pacientes.

Derecho a la privacidad y consentimiento informado. Los autores declaran que en este artículo no aparecen datos de pacientes.

\section{Financiación}

Fuente de financiación: Ministerio de Salud y Protección Social. Alianza CINETS.

\section{Conflictos de intereses}

Los autores declaran no tener ningún conflicto de intereses.

\section{Agradecimientos}

Agradecemos la contribución de las personas que, en carácter de representantes de expertos temáticos, usuarios, población blanco o grupos de interés, asistieron o participaron en las reuniones de socialización realizadas durante el desarrollo de la presente guía.

Agradecemos la participación de las instituciones, las asociaciones y las sociedades científicas, a través de sus representantes, por sus aportaciones y contribución en los diferentes procesos del desarrollo de la guía:

Asociación Colombiana de Medicina Interna (ACMI). Sociedad Colombiana de Cardiología y Cirugía Cardiovascular.

Asociación Colombiana de Endocrinología, Diabetes y Metabolismo.

Asociación Colombiana de Fisioterapia (ASCOFI).

Centro Colombiano de Nutrición Integral (CECNI). Centro Nacional de Investigación en Evidencia y Tecnologías en Salud (Alianza CINETS).

\section{Bibliografía}

1. Anderson TJ, Grégoire J, Hegele RA, Couture P, Mancini GBJ, McPherson R, et al. 2012 update of the Canadian Cardiovascular Society guidelines for the diagnosis and treatment of dyslipidemia for the prevention of cardiovascular disease in the adult. Can J Cardiol [Internet]. 2012;29(2):151-67 [citado 22 May 2013]. Disponible en: http://www.ncbi.nlm.nih.gov/pubmed/23351925.

2. Eckel RH, Jakicic JM, Ard JD, Miller NH, Hubbard VS, Nonas C A, et al. 2013 AHA/ACC Guideline on lifestyle management to reduce cardiovascular risk: a report of the American College of Cardiology/American Heart Association task force on practice guidelines. J Am Coll Cardiol [Internet]. Elsevier Ltd; 2013 [citado 12 Dic 2013]. Disponible en: http://www.ncbi.nlm.nih.gov/pubmed/24239922

3. Goff DC, Lloyd-Jones DM, Bennett G, O'Donnell CJ, Coady $\mathrm{S}$, Robinson J, et al. 2013 ACC/AHA Guideline on the assessment of cardiovascular risk: a report of the American College of Cardiology/American Heart Association Task Force on Practice Guidelines. J Am Coll Cardiol [Internet]. Elsevier Ltd; 2013 [citado 12 Dic 2013]. Disponible en: http://www.ncbi.nlm.nih.gov/pubmed/24239921

4. Jensen MD, Ryan DH, Apovian CM, Loria CM, Ard JD, Millen $\mathrm{BE}$, et al. $2013 \mathrm{AHA} / \mathrm{ACC} / \mathrm{TOS}$ Guideline for the management of overweight and obesity in adults: a report of the American College of Cardiology/American Heart Association Task Force on Practice Guidelines and The Obesity Society. J Am Coll Cardiol [Internet]. Elsevier Ltd; 2013 [citado 17 Dic 2013]. Disponible en: http://www.ncbi.nlm.nih.gov/pubmed/24239920 
5. Reiner Z, Catapano AL, de Backer G, Graham I, Taskinen MR, Wiklund O, et al. ESC/EAS Guidelines for the management of dyslipidaemias: the task force for the management of dyslipidaemias of the European Society of Cardiology (ESC) and the European Atherosclerosis Society (EAS). Eur Heart J [Internet]. 2011;32(14):1769-818 [citado 22 May 2013]. Disponible en: http://www.ncbi.nlm.nih.gov/pubmed/21712404.

6. Jun M, Foote C, Lv J, Neal B, Patel A, Nicholls SJ, et al. Effects of fibrates on cardiovascular outcomes: a systematic review and meta-analysis. Lancet [Internet]. 2010;375(9729):1875-84. Disponible en: http://www.ncbi.nlm.nih.gov/pubmed/20462635.

7. Abourbih S, Filion KB, Joseph L, Schiffrin EL, Rinfret S, Poirier $P$, et al. Effect of fibrates on lipid profiles and cardiovascular outcomes: a systematic review. Am J Med [Internet]. 2009;122(10), 962.e1-8. [citado 22 Nov 2013]. Disponible en: http://www.ncbi.nlm.nih.gov/pubmed/19698935.

8. Zhou Y-H, Ye X-F, Yu F-F, Zhang X, Qin Y-Y, Lu J, et al. Lipid management in the prevention of stroke: a meta-analysis of fibrates for stroke prevention. BMC Neurol [Internet]. 2013 [citado 22 Nov 2013].13:1. Disponible en: http:// www. pubmedcentral.nih.gov/articlerender. fcgi?artid=3554504 \&tool=pmcentrez\&rendertype $=$ Abstract

9. Sharma M, Ansari MT, Abou-Setta AM, Soares-Weiser K, Ooi TC, Sears $M$, et al. Systematic review: comparative effectiveness and harms of combination therapy and monotherapy for dyslipidemia. Ann Intern Med [Internet]. 2009;151(9):622-30 [citado 22 Nov 2013]. Disponible en: http://www.ncbi.nlm.nih.gov/pubmed/19884623.

10. Lavigne PM, Karas RH. The current state of niacin in cardiovascular disease prevention: a systematic review and meta-regression. J Am Coll Cardiol [Internet] Elsevier Inc;. 2013;61(4):440-6 [citado 28 Ago 2013]. Disponible en: http://www.ncbi.nlm.nih.gov/pubmed/23265337.

11. Duggal JK, Singh M, Attri N, Singh PP, Ahmed N, Pahwa S, et al. Effect of niacin therapy on cardiovascular outcomes in patients with coronary artery disease. J Cardiovasc Pharmacol Ther [Internet]. 2010;15(2):158-66 [citado 18 Sep 2013]. Disponible en: http://www.ncbi.nlm.nih.gov/pubmed/20208032.

12. Group HC. HPS2-THRIVE randomized placebo-controlled trial in 25673 high-risk patients of ER niacin/laropiprant: trial design, pre-specified muscle and liver outcomes, and reasons for stopping study treatment. Eur Heart J [Internet]. 2013;34(17):1279-91 [citado 18 Sep 2013] Disponible en: http://www.pubmedcentral.nih.gov/articlerender.fcgi?artid= $3640201 \&$ tool=pmcentrez\&rendertype=Abstract .

13. Rizos E, Ntzani E, Kostapanos MS. Association between omega-3 fatty acid. JAMA. 2013;308:1024-33.

14. Roncaglioni MC, Tombesi M, Avanzini F, Barlera S, Caimi V, Longoni P, et al. N-3 Fatty acids in patients with multiple cardiovascular risk factors. N Engl J Med [Internet]. 2013;368(19):1800-8 [citado 6 Ago 2013]. Disponible en: http://www.ncbi.nlm.nih.gov/pubmed/23656645.
15. Chowdhury R, Stevens S, Gorman D, Ward H, Johnson L. Association between fish consumption, long chain omega 3 fatty acids, and risk of cerebrovascular disease: systematic review and meta-analysis. BMJ. 2012;345(r):1-9.

16. Hooper L, Harrison R, Summerbell C, Moore H, Worthington $\mathrm{H}$. Omega 3 fatty acids for prevention and treatment of cardiovascular disease (Review). Cochrane Database Syst Rev. 2009;(4):1-193.

17. Pan A, Chen M, Chowdhury R, Sun Q, Campos H, Mozaffarian $D$, et al. a-Linolenic acid and risk of cardiovascular disease: a systematic. Am J Clin Nutr. 2012;96:1262-73.

18. Davidson MH, Donovan JM, Misir S, Jones MR. A 50-week extension study on the safety and efficacy of colesevelam in adults with primary hypercholesterolemia. Am J Cardiovasc Drugs [Internet]. 2010;10(5):305-14. Disponible en: http://www.ncbi.nlm.nih.gov/pubmed/20860413.

19. Gordon D, Knoke J, Probstfield J. High-density lipoprotein cholesterol and coronary heart disease in hypercholesterolemic men: the lipid research clinics coronary primary prevention trial. Circulation. 1986;74:1217-25.

20. Rifkind B. The lipid research clinics coronary primary prevention Trial. Drugs. 1986;31 Suppl 53-60.

21. Dorr AE, Gundersen K, Schneider JC, Spencer TW, Martin WB. Colestipol hydrochloride in hypercholesterolemic patientseffect on serum cholesterol and mortality. J Chronic Dis [Internet]. 1978;31(1):5-14 [citado 17 Mar 2014]. Disponible en: http://www.ncbi.nlm.nih.gov/pubmed/ 346598.

22. Insull W, Toth P, Mullican W, Hunninghake D, Burke S, Donovan JM, et al. Effectiveness of colesevelam hydrochloride in decreasing LDL cholesterol in patients with primary hypercholesterolemia: a 24-week randomized controlled trial. Mayo Clin Proc [Internet]. 2001;76(10):971-82 [citado 17 Mar 2014]. Disponible en: http://www.ncbi.nlm.nih.gov/pubmed/11605698.

23. Pandor A, Ara RM, Tumur I, Wilkinson AJ, Paisley S, Duenas $A$, et al. Ezetimibe monotherapy for cholesterol lowering in 2,722 people: systematic review and meta-analysis of randomized controlled trials. J Intern Med [Internet]. 2009;265(5):568-80 [citado 30 Ago 2013] Disponible en: http://www.ncbi.nlm.nih.gov/pubmed/19141093.

24. Mikhailidis DP, Lawson RW, McCormick A-L, Sibbring GC, Tershakovec AM, Davies GM, et al. Comparative efficacy of the addition of ezetimibe to statin vs. statin titration in patients with hypercholesterolaemia: systematic review and meta-analysis. Curr Med Res Opin [Internet]. 2011;27(6):1191-210 [citado 2 Sep 2013] Disponible en: http://www.ncbi.nlm.nih.gov/pubmed/21473671.

25. Blazing MA, Giugliano RP, Cannon CP, Musliner TA, Tershakovec AM, White JA, et al. Evaluating cardiovascular event reduction with ezetimibe as an adjunct to simvastatin in 18,144 patients after acute coronary syndromes: final baseline characteristics of the IMPROVE-IT study population. Am Heart J. 2014;168:20512.e1. 